\title{
Discussing Depression with Vietnamese American Patients
}

\author{
Tonya L. Fancher · Hendry Ton · Oanh Le Meyer · \\ Thuan Ho · Debora A. Paterniti
}

Published online: 26 February 2009

(C) The Author(s) 2009. This article is published with open access at Springerlink.com

\begin{abstract}
Background Asian patients preferentially seek mental health care from their primary care providers but are unlikely to receive it. Primary care providers need culturally-informed strategies for addressing stigmatizing illnesses. Methods 11 Vietnamese American community members participated in semi-structured interviews. Interviews were audio-taped and transcribed. The grounded theory approach was used for qualitative coding and thematic analysis. Results Vietnamese community members describe experiences with depression under four themes: (1) Stigma and face; (2) Social functioning and the role of the family; (3) Traditional healing and beliefs about medications; and (4) Language and culture. Based on this data,
\end{abstract}

This project was presented in part as a poster presentation at the Society of General Internal Medicine National meeting in 2007 and the Society of General Internal Medicine California Regional Meeting in 2007.

T. L. Fancher $(\bowtie) \cdot$ D. A. Paterniti

Department of Internal Medicine, University of California Davis Medical Center, 3100 Patient Support Services Building,

Sacramento, CA 95718, USA

e-mail: TLFancher@UCDavis.edu

H. Ton

Department of Psychiatry, University of California Davis

Medical Center, Sacramento, CA, USA

O. Le Meyer

Department of Psychology, University of California Davis,

Davis, CA, USA

T. Ho

Howard University College of Medicine, Washington, DC, USA

D. A. Paterniti

Department of Sociology, University of California Davis, Davis, DC, USA we offer suggestions for improving culturally-informed care for Vietnamese Americans. Disucssion Our study adds to the research aimed at improving communication and health care relationships between physicians and Vietnamese American patients. Physicians should learn to tailor their interviewing style to the increasingly diverse patient population.

Keywords Communication - Depression · Primary care . Vietnamese American

\section{Background}

Asian American patients in mainstream mental health systems have greater premature dropout rates, shorter duration of treatment, fewer positive outcomes, and less satisfaction with care compared to White American patients [1,2]. As such, Asian patients preferentially seek mental health care from their primary care providers but are unlikely to receive it [3,4]. Over 13 million Asian Americans representing over 35 different subgroups live in the U.S. [5]; Vietnamese Americans are the fourth largest Asian subgroup (following Chinese, Asian Indian and Filipino). Among Vietnamese Americans, experiences of war trauma, post-traumatic stress disorder and resettlement challenges contribute to the alarming rates of depression with estimates ranging from 8 to $50 \%$ [6, 7]. Because culture affects mental illness with respect to the way patients describe symptoms, the meaning they impart to their illness, and how they make sense of the experience of illness [8], primary care providers require strategies for addressing the mental health needs of ethnic minorities. We conducted a series of in-depth interviews with Vietnamese community members to understand the community 
perspective on depression and develop strategies for culturally-informed mental health care for non-mental health providers.

\section{Methods}

\section{Participants and Data Collection}

Interviews were conducted with Vietnamese mental health experts, patients with depression, and their family members identified first by local experts in the mental health field and then through snowball sampling [9]. Interviews were conducted in either Vietnamese or English by a interviewer who was a native Vietnamese speaker (TH) and trained by the sociologist (DAP), internist (TLF) and cultural psychiatrist (HT). The interviewer used a set of guiding questions and probes, developed through extensive literature review and in discussion with the multi-disciplinary team. All interviews were audio-recorded and then transcribed verbatim for analysis.

Eleven interviews with first or second-generation Vietnamese immigrants were conducted in Sacramento, California between November 2005 and February 2006. Six women and five men, ranging in age from 30 to 65 years, were interviewed. All participants had experience with mental illness as patients, as family members or friends of patients with mental illness, or as mental health care providers. All interviews were offered in Vietnamese or English.

\section{Analysis}

The grounded theory approach to data analysis was employed [10]. Transcripts were reviewed independently by research team members (TLF, TH, DAP) for predominant and recurring themes [9]. Team members met on several occasions to discuss emergent themes and patterns and their categorization, resolving disagreement by consensus and consideration of clinical relevance for depression treatment and management. Each set of excerpts was reviewed by the cooperative research team, and then themes and sub-codes were characterized. From these common themes and patterns, the team developed specific coding categories to be applied to all transcripts.

The research was approved by the University of California Davis Institutional Review Board.

\section{Results}

Our Vietnamese participants described experiences with depression and encounters with primary care systems and providers under four themes: (1) Stigma and face; (2)
Social functioning and the role of the family; (3) Traditional healing and beliefs about medications; and (4) Language and culture.

Stigma and Face

“... it seems that the term [depression] is too harsh at this point for [the patient]. You can use something similar but a little lighter ... some people associate every [mental health] thing with crazy."

Patients and families may deny signs and symptoms of mental illness to preserve their public appearance and save face for themselves and their family [11]. Others in the ethnic community may perceive mental illness as a reflection of poor moral character, spiritual weakness, or improper upbringing by the family [12]. Reflections on personal experiences in Vietnam where mental illness was associated with institutionalization or imprisonment shape perceptions of mental illness.

Social Functioning and the Role of the Family

“... the Vietnamese community...treats these problems within the home and/or kinship network first. And I do not think they look at depression as such [a problem]. It might be: this person is not getting work... then they would attack those issues one at a time in the kinship network, aunts and uncles and cousins and very good friends trying to help out."

Consistent with the literature on interdependence in many Asian cultures [13], our participants emphasized the importance of including the family in medical care decisionmaking and in thinking about depression as a disruption of social group functioning rather than as an individual mental health problem. Distrust or stigma regarding psychiatric services often leads to intensive family involvement in lieu of formal treatment [12]. Even when psychiatric treatment is identified as appropriate and is sought, family members may be concerned that others may be judgmental or may themselves feel that a patients' mental illness is a result of inadequate family support. The struggles between traditional and new cultural standards surrounding the family provider, familial hierarchy, and social roles create additional stresses that impact mental well-being [14].

Traditional Healing and Beliefs about Medications

"Vietnamese, especially immigrants and/or those who have some experience living abroad from other 
countries and are coming here, tend... to value Eastern medicine if not more than equally or at least have some belief in its uses... they want to seek that sort of medication first, because it is seen as more organic and something that they are familiar with and something they grew up [with]"

Because Vietnamese Americans may believe that mental illness stems from metaphysical imbalances, they may use traditional methods before coming to Western providers [15]. Vietnamese cultural beliefs about medications include the following: (1) Western medications work quickly and have many strong side effects; (2) Being on Western medications for a long time can be harmful to the body; and (3) Vietnamese tolerate less medication than Caucasians and Western trained physicians may not realize this. Vietnamese beliefs can conflict with current understandings of how antidepressants work and can, therefore, impede long-term adherence to anti-depressant medications.

\section{Language and Culture}

"I think that people will get help...[from] a Vietnamese doctor so they can explain their problems... That way, they can [give a] full explanation to the doctor [rather] than go ...to the hospital where they speak to the doctor ... [who does not speak]...in their language."

Participants felt that non-Vietnamese providers were unable to understand the nuances of their culture that include respect for authority, need for smooth relationships and traditional interdependent family relationships. Concerns about communication difficulties were common; one participant noted: "I think we need to get professional help. But the professional help cannot come from an American doctor, because they need to understand our culture, how to reach into the people who bottle up like that. But if they think the American way and expect the patient to act the American way, then we go nowhere."

\section{Discussion}

When therapy is culturally responsive, patients participate more in treatment and experience better outcomes [12]. Lower levels of stigma in primary care settings makes them ideal settings for addressing the mental health needs of ethnic minorities [16]. To ensure more culturally informed care for Vietnamese patients, providers should aim to minimize stigma and help patients find face-saving communication strategies (Table 1). Because patients may not want to question or disagree with the physician, providers should use face-saving strategies to ask difficult questions, e.g. "Many of my patients express the positive and negative effects of these medications, and I have found the added information to be very helpful in customizing the treatment to your particular health issues." This approach allows the patient to express concerns while also preserving the doctor's role as an expert. Providers should also aim to address social functioning and family roles. If acceptable to the patient, the provider can actively engage the family in the presence of the patient and help to rebuild their social networks. Care must be taken when psychoeducating families about mental illness, as it may exacerbate their feelings of stigma and/or failure. After visiting their physicians, many patients will consult with family

Table 1 Strategies to improve depression communication with Vietnamese American patients

To minimize stigma and help patients find face-saving communication strategies

- Explore the somatic complaints of depression (insomnia, poor appetite) before exploring the emotional components.

- Replace particularly stigmatizing terms e.g. "mental illness," "depression," and "suicide” with "feelings," "stress," and "giving-up."

To address social functioning and family roles

- Ask the patient about their role in the family, the ways their illness affects life as experienced by the family and to identify key family decision makers.

- Encourage the family to recount their efforts to help the patient.

- Validate the family's efforts and reassure them that one would expect even the "best" of families would need expert help to treat the patient's condition.

To incorporate traditional beliefs into the treatment plan

- Use stress to explain depression: "You've been through a lot of stress in your life, more so than most people. The stress has worn you down, and that is why you have problems with sleep, concentration, energy, and even sadness. Even the strongest of people would be affected by the stresses that you have experienced."

- Consider starting an anti-depressant that has an immediate effect (e.g. sedating antidepressant for insomnia or a combination of a sedative and an antidepressant) at a very low dose and increase the dosage slowly.

To optimize language concordance and cultural understanding

- Explore local or telehealth sources of bilingual and bicultural staff. 
members for assistance with the physician's instructions. Traditionally, the elders in the family fulfill this role but younger more acculturated family members are increasingly taking on this role [17]. While education about the biomedical causes of mental illness alleviates stigma for some Westernized patients, it may work less well for Vietnamese patients who have less exposure to the biomedical conceptual framework. Vietnamese patients will value efforts to incorporate traditional beliefs into the treatment plan with an explanation of depression as an imbalance in chemicals that affects both the brain and body. If a specific course of action is not prescribed or if the medications do not work quickly, patients may believe the medications are ineffective and the provider and treatment strategy then loses credibility. To optimize language concordance and cultural understanding bilingual and bicultural staff are essential members of the healthcare team [18]. Children and family members should not be relied upon as interpreters. Patients appreciate a provider who expresses an interest in their background and see this as a foundation for a trusting relationship.

Acknowledgements Dr. Fancher, Dr. Paterniti and Ms. Meyer are supported by the Asian American Center on Disparities Research, NIMH Grant \# MH073511-01A2. Drs. Fancher and Paterniti and Mr. Ho received funding for a University of California Health System grant. The authors wish to thank Richard Kravitz MD for his critical review and comments on the manuscript.

Open Access This article is distributed under the terms of the Creative Commons Attribution Noncommercial License which permits any noncommercial use, distribution, and reproduction in any medium, provided the original author(s) and source are credited.

\section{References}

1. Zane NWS, Enomoto K, Chun C. Treatment outcomes of Asian and White American clients in outpatient therapy. J Community Psychol. 1994;22:177-91. doi:10.1002/1520-6629(199404)22:2 $<177::$ AID-JCOP2290220212>3.0.CO;2-7.

2. Nguyen QCX, Anderson LP. Vietnamese Americans' attitudes towards seeking mental health services: relation to cultural variables. J Community Psychol. 2005;33(2):213-31. doi:10.1002/ jcop.20039.
3. Chung H, Nguyen D, Gany F. Initial behavioral health assessment of Asian Americans. Part 1. Key principles. West J Med. 2002;176(4):233-6.

4. Sorkin, D., et al. Self-reported health status of Vietnamese and non-hispanic white older adults in California. J Am Geriatr Soc. 2008;56(8):1543-8.

5. Census, U.S. Projections of the resident population by race, hispanic origin, and nativity: middle series, 2001 to 2005. In: BUS Census, editor. Washington, DC.: US Government Printing Office; 2000.

6. Mollica RF, et al. The dose-effect relationships between torture and psychiatric symptoms in Vietnamese ex-political detainees and a comparison group. J Nerv Ment Dis. 1998;186(9):543-53. doi:10.1097/00005053-199809000-00005.

7. Hinton L, et al. A survey of depressive symptoms among Vietnamese-American men in three locales: prevalence and correlates. J Nerv Ment Dis. 1998;186(11):677-83. doi:10.1097/00005053199811000-00003.

8. Kleinman A, Eisenberg LE, Good BJ. Culture, illness, and care: clinical lessons from anthropological and cross-cultural research. Ann Intern Med. 1978;99:25-58.

9. Denzin NK, Lincoln YS. The handbook of qualitative research. 2nd ed. Thousand Oaks: Sage Publications; 2000.

10. Strauss AL, Corbin J. Basics of qualitative research: techniques and procedures for developing grounded theory. 2nd ed. Thousand Oaks: Sage Publications; 1998.

11. Kim BSK, Atkinson DR, Umemoto D. Asian cultural values and the counseling process: current knowledge and directions for future research. Couns Psychol. 2001;29(4):570-603. doi:10.1177/ 0011000001294006.

12. Sue DW, Sue D. Counseling the culturally different: theory and practice. 3rd ed. Hoboken, NJ, US: John Wiley \& Sons Inc; 1999. 368.

13. Markus HR, Kitayama S. Culture and the self: implications for cognition, emotion and motivation. Psychol Rev. 1991;98:224-53. doi:10.1037/0033-295X.98.2.224.

14. Uba L. Asian Americans personality patterns, identity, and mental health. The Guilford Press: New York; 1994. p. 26-225.

15. Lin K-M, Cheung F. Mental health issues for Asian Americans. Psychiatr Serv (Washington, D.C.). 1999;50(6):774-80.

16. Schraufnagel TJ, et al. Treating minority patients with depression and anxiety: what does evidence tell us? Gen Hosp Psychiatry. 2006;28:27-36. doi:10.1016/j.genhosppsych.2005.07.002.

17. Kim BSK, et al. A qualitative study of adaptation experiences of 1.5 generation Asian Americans. Cult Div Ethn Minor Psychol. 2003;9:156-70. doi:10.1037/1099-9809.9.2.156.

18. Ngo-Metzger Q, et al. Providing high-quality care for limited English proficient patients: the importance of language concordance and interpreter use. J Gen Intern Med. 2007;22(Suppl 2): 324-30. doi:10.1007/s11606-007-0340-z. 